\title{
Estudio de investigaciones sobre el neuromarketing en turismo
}

\author{
Research study on neuromarketing in tourism
}

\author{
Soima Rosa Méndez Lazo. ${ }^{1}$,Yasser Vázquez Alfonso. ${ }^{2}$, Sacha Lazo del Vallín. ${ }^{3}$ \& \\ Yolanda Tatiana Carrasco Ruano. ${ }^{4}$
}

DOI: https://doi.org/10.33262/visionariodigital.v5i4.1876

\begin{abstract}
A bibliometric analysis was carried out on neuromarketing, given the relevance of scientific publications on the subject and the diversity of theories of its conceptualization. As indicators, the productivity of the authors, the collaboration in the publications, the descriptors studied, the years of publication and the distribution of the research according to journals or branches of science were considered. Multivariate statistical methods were used such as Cluster Analysis with the corresponding algorithms and the New Zealand software Weka 3.9.5. From retrospective observation, the contingency between qualitative variables such as psychocognitive processes, neuroimaging techniques and their scope in the marketing of sectors of the economy such as tourism was interpreted, under the paradigm of scientific neuroethics, given its application in customer behavior investigations. To determine the regression between two or more variables, given the adjustments between the obtained functions and the point cloud, goodness of fit methods were applied. As main results, the functional dependence among the variables studied prevailed and to a lesser extent the random or partial statistical correlation. The most significant were the galvanic skin response biometry technique, consumer behavior, as well as the psychological influence of brands in the purchase process and the placebo effect. Its comprehensive analysis at the business level allows increasing the effectiveness
\end{abstract}

\footnotetext{
${ }^{1}$ Licenciada en Turismo. Profesor instructor. Facultad de Turismo. Universidad de la Habana soimita94@gmail.com, https://orcid.org/0000-0001-7470-4404

${ }^{2}$ Doctor en Ciencia Veterinaria. Profesor Titular. Facultad de Turismo. Universidad de la Habana.yafos1@gmail.com, https://orcid.org/0000-0002-4074-0711

${ }^{3}$ Máster en Ciencias Médicas y Médico Especialista en Imagenología. Profesor asistente e Investigador auxiliar. Instituto de Gastroenterología. delvallin@infomed.sld.cu, https://orcid.org/0000-0002-4001-9596

${ }^{4}$ Ciencia digital Editorial, tcarrascor@hotmail.com, https://orcid.org /0000-0002-5294-8202
} 
of advertising campaigns and commercial management in line with the sustainable development of the tourism economy.

Keywords: neuromarketing; bibliometrics; consumer behavior; neuroimaging techniques; Cuba

\section{Resumen}

Se realizó un análisis bibliométrico sobre el neuromarketing, dada la relevancia de las publicaciones científicas sobre el tema y la diversidad de teorías de su conceptualización. Como indicadores se consideraron la productividad de los autores, la colaboración en las publicaciones, los descriptores estudiados, los años de publicación y la distribución de las investigaciones según revistas o ramas de la ciencia. Se emplearon métodos estadísticos multivariados como el Análisis de Clúster con los algoritmos correspondientes y el software neozelandés Weka 3.9.5. Desde la observación retrospectiva, se interpretó la contingencia entre variables cualitativas como los procesos psicocognitivos, las técnicas de obtención de neuroimagen y su alcance en la mercadotecnia de sectores de la economía como el turismo, bajo el paradigma de la neuroética científica, dada su aplicación en las investigaciones de comportamiento del cliente. Para determinar la regresión entre dos o más variables, dado los ajustes entre las funciones obtenidas y la nube de puntos, se aplicaron métodos de bondad de ajuste. Como resultados principales, primó la dependencia funcional entre las variables estudiadas y en menor medida la correlación aleatoria o parcial estadística. Las de mayor significación fueron la técnica de biometría respuesta galvánica de la piel, el comportamiento del consumidor, así como la influencia psicológica de las marcas en el proceso de compra y el efecto placebo. Su análisis integral a nivel empresarial permite incrementar la efectividad de las campañas publicitarias y la gestión comercial en consonancia con el desarrollo sostenible de la economía del turismo.

Palabras claves: neuromarketing; bibliometría; comportamiento del consumidor; técnicas de neuroimagen; Cuba.

\section{Introducción}

El análisis bibliométrico es un componente cardinal de los estudios sociales de la ciencia (Fajardo-Egües, 2016). El aumento de la producción científica en periodos recientes, unido a su provisión bibliográfica en bases de datos automatizadas, ha impulsado el uso de la bibliometría y la creación de indicadores cuantitativos para la medición y certificación de las deducciones científicos y tecnológicos (Victoria, Arjona, \& Repiso, 2015).

El turismo es un sector dinamizador de la economía en Cuba y en el mundo. Las investigaciones de mercado actuales requieren de creatividad, gestión del conocimiento y la innovación. Adentrarse en el subconsciente del consumidor de la oferta turística, permite diseñar perfiles de clientes más precisos y eficaces, orientados a lo que siente y 
piensa la persona, lo que no tiene que coincidir necesariamente lo que verbaliza en métodos tradicionales de recolección de datos como entrevistas y encuestas. Autores como Nonone \& Regalado (2019), apuntan que es precisamente en el subconsciente del consumidor, donde pretende llegar el neuromarketing, resultado de la fusión de las neurociencias y el marketing. Los resultados de la aplicación de técnicas de neuroimagen no invasivas para el usuario, pueden ser interpretados, representados gráficamente y establecer tendencias y patrones de comportamiento para predecir su conducta en base a los intereses propios de la mercadotecnia. Las prestaciones del neuromarketing en países como Estados Unidos, Colombia, Alemania e Inglaterra han sido palpables en ramas de estudio de la hotelería, la restauración, la gestión de destinos turísticos inteligentes y sostenibles de mano de la innovación tecnológica, el desarrollo de productos y el comercio electrónico (redes sociales, email marketing), como variable transversal.

El objetivo general de esta investigación fue realizar un análisis bibliométrico multivariado de la producción científica internacional del neuromarketing en el turismo y sus proyecciones para Cuba.

\section{Materiales y métodos}

Se realizó un estudio multivariado de la producción científica internacional sobre el neuromarketing. Se utilizaron las publicaciones registradas en bases científicas como Web of Science, Scopus, ScienceDirect y Scielo, durante el periodo 2005-2021. Entre los documentos revisados se encontraron tesis de diploma, maestría y doctorado, libros, memorias de eventos y artículos en revistas especializadas.

Se aplicó una batería de indicadores bibliométricos para la evaluación de aspectos del neuromarketing partir de 5 bloques de interés: cerebro humano, técnicas de neuroimagen, tipología de neuromarketing o clasificación según los sentidos estimulados, parámetros de estudio y aspectos de turismo, desglosados en sus respectivos descriptores, como se muestra en la tabla 1. Fue integrado lo cualitativo y cuantitativo, se realizó un análisis estadístico multivariado y se elaboró un informe final con los resultados arrojados.

Tabla 1 Características de las variables

\begin{tabular}{|c|c|c|c|c|}
\hline No & Variables & Dimensiones & Tipo & Escala \\
\hline 1 & Estructura y funciones del cerebro (EFC) & Cerebro humano & Cualitativa & Nominal \\
\hline 2 & Teoria del cerebro triuno (TCT) & Cerebro humano & Cualitativa & Nominal \\
\hline 3 & Resonancia magnética (RM) & Neuroimagen & Cualitativa & Nominal \\
\hline 4 & Electroencefalografia (EF) & Neuroimagen & Cualitativa & Nominal \\
\hline 5 & Electrocardiograma (ET) & Neuroimagen & Cualitativa & Nominal \\
\hline 6 & Electromiografia (EM) & Neuroimagen & Cualitativa & Nominal \\
\hline 7 & Evetracking (ETR) & Neuroimagen & Cualitativa & Nominal \\
\hline 8 & Pupilometria (PM) & Neuroimagen & Cualitativa & Nominal \\
\hline 9 & Codificación facial (CF) & Neuroimagen & Cualitativa & Nominal \\
\hline 10 & Respuesta galvánica de la piel (RGP) & Neuroimagen & Cualitativa & Nominal \\
\hline 11 & Test de Asociación Implicita (TAI) & Neuroimagen & Cualitativa & Nominal \\
\hline 12 & Neuromarketing visual (NV) & Tipologia & Cualitativa & Nominal \\
\hline 13 & Neuromarketing auditivo (NA) & Tipologia & Cualitativa & Nominal \\
\hline 14 & Neuromarketing kinestésico (NK) & Tipologia & Cualitativa & Nominal \\
\hline 15 & Neurotrangmigores (NT) & Parámetro estudio & Cualitativa & Nominal \\
\hline 16 & Comportamiento del consumidor (CC) & Parámetro estudio & Cualitativa & Nominal \\
\hline 17 & Proceso de compra (PC) & Parámetro estudio & Cualitativa & Nominal \\
\hline 18 & Atención (A) & Cerebro humano & Cualitativa & Nominal \\
\hline 19 & Emoción (E) & Cerebro humano & Cualitativa & Nominal \\
\hline 20 & Memoria (M) & Cerebro humano & Cualitativa & Nominal \\
\hline 21 & Teoria del Color (TC) & Cerebro humano & Cualitativa & Nominal \\
\hline 22 & Sistema de Recompensa del Cerebro (SRC) & Parámetro estudio & Cualitativa & Nominal \\
\hline
\end{tabular}


Tabla 1 Características de las variables (continuación)

\begin{tabular}{|c|c|c|c|c|}
\hline 23 & Efecto Placebo (EP) & Parámetro estudio & Cualitativa & Nominal \\
\hline 24 & Funcionamiento de la mente (FM) & Parámetro estudio & Cualitativa & Nominal \\
\hline 25 & Marcador somático (MS) & Parámetro estudio & Cualitativa & Nominal \\
\hline 26 & Redes neuronales (RN) & Parámetro estudio & Cualitativa & Nominal \\
\hline 27 & Etica (ETC) & Neuroimagen & Cualitativa & Nominal \\
\hline 28 & Publicidad y marca (PUB-M) & Aspecto de turismo & Cualitativa & Nominal \\
\hline 29 & Hoteleria (HOT) & Aspecto de turismo & Cualitativa & Nominal \\
\hline 30 & Restauración (A+B) & Aspecto de turismo & Cualitativa & Nominal \\
\hline 31 & Destinos y agencias de viajes (DYV) & Aspecto de turismo & Cualitativa & Nominal \\
\hline 32 & E-commerce (ECOM) & Aspecto de turismo & Cualitativa & Nominal \\
\hline 33 & Redes sociales (RS) & Aspecto de turismo & Cualitativa & Nominal \\
\hline 34 & Email Marketing (EM) & Aspecto de turismo & Cualitativa & Nominal \\
\hline 35 & Desarrollo de productos (DP) & Aspecto de turismo & Cualitativa & Nominal \\
\hline 36 & Comercio interior v Merchandising (CIMCH) & Assecto de turismo & Cualitativa & Nominal
\end{tabular}

Fuente: Elaboración propia

\section{Análisis estadístico}

Se realizó un muestreo aleatorio probabilístico para una población finita de 73 artículos de diversos países. Su cálculo se basó en una probabilidad de ocurrencia de 0,5; error máximo de estimación de 0,04 y confianza del 97,5\%. Se empleó la siguiente fórmula 1 utilizada por Moráguez (2011) para investigaciones en ciencias sociales, dando como resultado una muestra de 65 artículos:

$$
\mathrm{n}=\frac{\mathrm{n}_{\mathrm{o}}}{1+\frac{\mathrm{n}_{\mathrm{o}}}{\mathrm{N}}} \text { donde: } \mathrm{n}_{\mathrm{o}}=p^{*}(1-p)^{*}\left(\frac{\mathrm{Z}\left(1-\frac{\alpha}{2}\right)}{\mathrm{d}}\right)^{2}
$$

Donde:

$\mathrm{n}_{\mathrm{o}}$ : tamaño de muestra preliminar.

N: tamaño de la población.

n: tamaño de muestra con corrección para población finita.

p: proporción de éxito en el análisis que se realiza.

Los datos fueron recopilados en una base de datos de Microsoft Excel 2016. Se realizó un análisis multivariado para la evaluación simultánea de conjuntos de datos y poder explicar las relaciones existentes entre las variables vinculadas a la muestra. Como el tipo de variables que se utilizaron en el estudio se clasifican en cualitativa en forma binaria y en escala nominal, el método estadístico que se emplea el Análisis de Clúster para establecer grupos de individuos, unificando dentro de un mismo grupo a aquellos elementos que tengan características similares. En los métodos de clasificación juega un rol importante el índice de similitud o distancia utilizada pues de ello depende en gran medida que los resultados finales tengan la mayor confiabilidad posible (MIRANDA, 1997). Como las variables son binarias según (Vilà et.al, 2014), se utiliza la métrica de Jaccard que precisa un porcentaje calculado como \# de variables en que dos individuos coinciden / \# de variables analizadas. En la aplicación del Análisis de Clúster se utilizó el software SSPS versión 25.0.

Para comprobar si la clasificación a partir del Análisis de Clúster es correcta, se hace uso de los algoritmos OneR, BayesNet, RandomTree, NaiveBayes, RandomForest, 
MultilayerPerceptron, HoeffdingTree y SMO del software weka 3.9.5, desarrollado por la Universidad de Waikato, Nueva Zelanda en el 2020.

En la valoración de los resultados de esos algoritmos, se aplican una serie de indicadores de bondad de ajuste según (Martínez, 2018) y estos son:

- Índice Kappa de Cohen: Índice de bondad de ajuste del modelo completo. Se entiende que el modelo es apropiado si este valor supera el valor 0.7.

- Error absoluto medio: Estadístico que indica el error de estimación cometido. Valores más bajos indican que el modelo es más apropiado.

- Porcentaje del área bajo la curva ROC: La curva ROC indica, en un eje de abscisas y ordenadas, la relación entre la sensibilidad (verdaderos positivos entre el total de positivos) y especificidad (verdaderos negativos entre el total de negativos) del modelo de clasificación establecido. Se establece una curva para cada categoría, en relación con el resto de categorías, indicando la capacidad del modelo para detectar casos pertenecientes a esa categoría de la variable criterio. Este porcentaje nos indica, por lo tanto, la precisión que tiene el modelo para identificar correctamente a los sujetos de un grupo, teniendo en cuenta tanto el porcentaje de acierto en la detección de esa categoría y el porcentaje de desaciertos al identificar a sujetos de esa categoría. Si el valor de la curva ROC en los algoritmos es cercano a 1, indica que se acercan al clasificador perfecto que serían los que tienen menor cantidad de errores al clasificar.

- Matriz de confusión: Tabla de contingencia que relaciona la clasificación dada por el modelo con el valor real que alcanza el sujeto en la variable criterio. Los valores que están en la diagonal principal son los casos clasificados correctamente.

- Precisión: Porcentaje de instancias bien clasificadas de entre todas las seleccionadas como positivas.

Para determinar un buen clasificador se debe considerar que este proporcione clasificaciones correctas (exactitud), se define como la probabilidad con la que clasifica de manera adecuada un caso seleccionado al azar, (Kohavi,1995). También se puede determinar cómo el número de casos clasificados de manera adecuada entre el número total de elementos (ver fórmula 1).

\#de-casos-clasificados-adecuadamente

Exactitud $=$

\#total-de-casos

Otro algoritmo del software weka que se aplicó fue el A priori de (Srikant y Agrawal, 1996), siendo este el primer algoritmo para la extracción de reglas de asociación de manera satisfactoria. Martínez (2018), plantea que el algoritmo A priori, establece las reglas teniendo en cuenta el soporte de los datos a la regla y la confianza de la propia regla. El soporte se refiere al número de instancias que están incluidas en la regla, y la confianza al porcentaje de instancias, de entre el total del soporte, que cumplen la regla, es decir, el número de casos que predice la regla correctamente (ver fórmula 2). 
soporte $(A \Rightarrow B)=P(A \cap B)$ confianza $(A \Rightarrow B)=P(B / A)=\frac{P(A \cap B)}{P(A)}$.

Pérez (2018), describe una serie de indicadores para valorar las reglas de asociación generadas por el algoritmo A priori y consisten en:

- MetricType: estadístico empleado para la toma de decisiones sobre la generación de las reglas:

a. Confidence: se generan las $\mathrm{n}$ reglas soportadas con un mayor índice de confianza.

b. Lift: se generan las $\mathrm{n}$ reglas soportadas que tengan un valor mayor del índice de confianza dividido entre el número de casos cubiertos por la norma. Este índice es independiente de la tasa de soporte. Las asociaciones con un lift igual o muy próximo a uno nos indica que no existe ninguna asociación entre los productos y que su presencia se debe al puro azar, si el lift es menor a uno nos indica que los atributos de la asociación guardan una relación negativa (la presencia de X implica la ausencia de Y) y si el lift es superior a uno nos indica que los atributos de la asociación guardan una relación positiva (la presencia de X implica la presencia de Y).

c. Leverage: Se generan las $\mathrm{n}$ reglas soportadas de las $\mathrm{n}$ diferencias máximas entre el porcentaje de confianza y el porcentaje esperado si existiera independencia entre la regla y el soporte.

d. Conviction: Se generan las n reglas soportadas a partir de los n valores máximos alcanzados en el cálculo de una probabilidad condicionada entre la regla y el soporte.

\section{Resultados y discusión}

En la figura 1 se puede observar el proceso de integración los autores hasta quedar, a partir de la distancia 18, formado por 8 clúster, el número 1 es el que más autores tiene para un total de 56 autores (Ariely \& Berns, Fernández, Murphy, Illes \& Reiner, Renvoisé \& Morin, Tichindelean \& Cetina, Suárez Lugo, Carreño \& Riay, Gang, Lin, Qi \& Yan, Tosun, Ozdemir \& Cubuk, Lee, Broderick, \& Chamberlain, de Oliveira \& Giraldi, Ćosić, Solomón, Pardo \& Saurit , Sánchez,Gómez \& Niquepa Coca, Quintero \& Martínez, Espinosa \& Díaz, Salas, Babilony, Augusto, Elias \& Agüero, Victoria, Arjona \& Repisa, Canales, Agarval \& Dutta, Ouazzani, Benlafkih et al., Baraybar-Fernández et al., Slijepčević, Popović \& Radojević, BOSAK, Johnson \& Ghuman, Giudici, Dettori \& Caboni, Madan \& Popli, Penney, Rojas, Caicedo \& Cudris, Lewis \& Bridger, Reinson, Dragolea \& Cotîrlea, Dițoiu \& Căruntu, Vidal \& Murillo, Cohen, Prayag \& Moital, Georges, Bayle \& Badoc, Acosta, Jiménez \& Salas, Smykova, Kazybaeva \& Tkhorikov, Bastiaansen et al., de la Serna et al., Braidot, Roth, Madan \& Popli, Solnais, Sánchez \& Ibáñez, Arroyo, Malfitano, Arteaga \& Romano, Al Abbas, Chen \& Saberi, Javor et al., Plassmann \& Weber, Branislav, Onay, Muñoz), el clúster 2 (Peréz yConstantinescu et al.), el clúster 3( Camarero), el clúster 4 (Koc\&Boz y Ranisav\& Branislav), el clúster 
5(Canepa), el clúster 6 (Slijepčević, Popović \& Radojević), el clúster 7(Benitez) y el clúster 8 (Stanton, Sinnott\&Huettel).

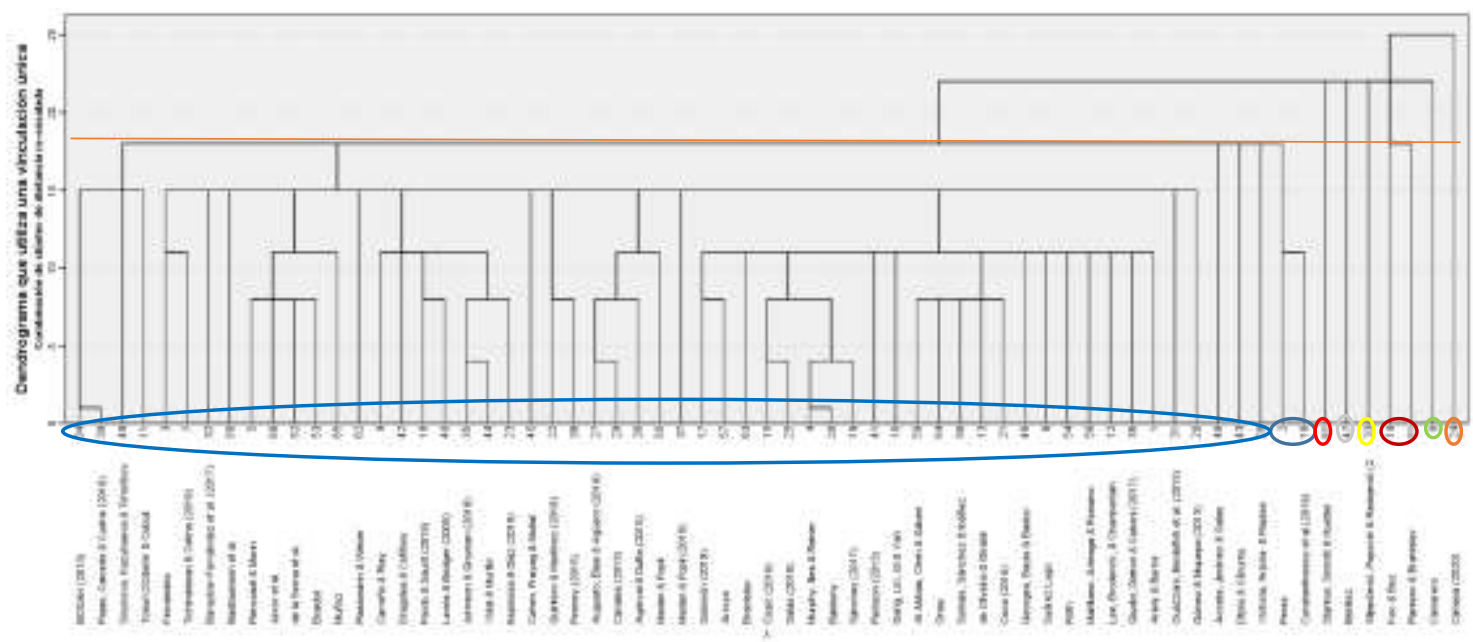

Figura 1. Gráfico del Clúster de los autores.

Fuente: Elaboración propia

El agrupamiento mayoritario de los intelectuales se justifica básicamente por su nacionalidad (las de mayor peso específico fueron Colombia, Estados Unidos, Inglaterra y España), las que coinciden con aquellas sedes de las revistas internacionales, siendo las más frecuentes Psychology Press, Natural Review of Neuroscience, Journal of Consumer Psychology, Frontiers in Human Neuroscience y Psychology and Marketing y con las principales consultoras internacionales de neuromarketing. Los mismos tuvieron mayor representatividad en la referencia de los descriptores de neuromarketing abordados, desde los procesos pscicognitivos, el funcionamiento de la mente, los estímulos cerebrales y tipos de neuromarketing en base a los sentidos que son involucrados por la publicidad. La generalidad de los autores concuerda con aquellos más citados en las publicaciones científicas acerca del tema de estudio. Se resalta además que en el 1,5\% de las situaciones, hubo colaboración de autores de distinta ubicación geográfica.

Paralelo a lo anterior, Brenninkmeijer, Schneider, \& Woolgar (2019) indican que más de la mitad de las publicaciones sobre neuromarketing se hallan en la base Scopus, resultando Estados Unidos, Inglaterra, Alemania, Canadá y España los 5 países con mayores publicaciones en dicha base (citado en Caicedo, 2021).

En la tabla 2 se muestra los diferentes resultados obtenidos con la aplicación de los algoritmos de clasificación implementados en el software weka. De todos se lograron significativos resultados por lo que constituyen buenos clasificadores para el conjunto de datos que se utilizó. Se destacaron en sus resultados los algoritmos OneR, RandomTree, RandomForest, MultilayerPerceptron y SMO, los cuales mostraron mejores valores de exactitud, índice Kappa, Área curva ROC y precisión. 
Tabla 2. Resultados de los algoritmos de clasificación con el weka

\begin{tabular}{ccccc}
\hline Algoritmos & Exactitud & Índice Kappa & Precisión & $\begin{array}{c}\text { Área curva } \\
\text { ROC }\end{array}$ \\
\hline OneR & 1 & 1 & $100 \%$ & 1 \\
BayesNet & 0.97 & 0.89 & $96.92 \%$ & 1 \\
NaiveBayes & 0.98 & 0.94 & $98.46 \%$ & 1 \\
RandomTree & 1 & 1 & $100 \%$ & 1 \\
RandomForest & 1 & 1 & $100 \%$ & 1 \\
MultilayerPerceptron & 1 & 1 & $100 \%$ & 1 \\
HoeffdingTree & 0.98 & 0.94 & $98.46 \%$ & 1 \\
SMO & 1 & 1 & $100 \%$ & 1 \\
\hline
\end{tabular}

Fuente: Elaboración propia.

Como resultado de la aplicación del algoritmo A priori del software weka se obtuvieron las mejores reglas que se ilustran en la tabla 3 , donde se resalta los diferentes valores de los indicadores de Confidence, Lift, Lev y Conv por cada regla.

Tabla 3: Mejores reglas encontradas.

\begin{tabular}{ccccc}
\hline Rule & Confidence & Lift & Lev & Conv \\
\hline CC $=$ Si $60==>$ RGP=No 59 & 0.98 & 1.01 & 0.01 & 0.92 \\
CC=Si EP=No 57 ==> RGP=No 56 & 0.98 & 1.01 & 0.01 & 0.88 \\
CC=Si PUBM=Si 56 ==> RGP=No 55 & 0.98 & 1.01 & 0.01 & 0.86 \\
RGP=No PUBM=Si 56 ==> CC=Si 55 & 0.98 & 1.06 & 0.05 & 2.15 \\
EP=No 62 ==> RGP=No 60 & 0.97 & 1 & 0 & 0.64 \\
ETR=No 60 ==> RGP=No 58 & 0.97 & 1 & 0 & 0.92 \\
ETR=No 60 ==> EP=No 58 & 0.97 & 1.01 & 0.01 & 0.64 \\
PUBM=Si 58 ==> RGP=No 56 & 0.97 & 1 & 0 & 0.59 \\
PUBM=Si 58 ==> CC=Si 56 & 0.97 & 1.05 & 0.04 & 1.49 \\
ETR=No EP=No 58 ==> RGP=No 56 & 0.97 & 1 & 0 & 0.59 \\
\hline
\end{tabular}

Fuente: Elaboración propia.

Son significativos los valores del indicador Lift superiores a 1 en correlaciones como la respuesta galvánica de la piel (RGP) y comportamiento del consumidor, el efecto placebo y su uso en el neuromarketing, así como la influencia de la publicidad de marca en la percepción del cliente de los valores y atributos del producto o servicio turístico. La notoriedad de la variable RGP, corresponde a su relevancia para el marketing. Su tecnología se basa en la monitorización de los cambios en la conductividad eléctrica de la piel, mediante las glándulas sudoríparas que reflejan la intensidad del estado emocional del consumidor, lo que explica tal correlación. Su base científica radica en que la respuesta emocional del cerebro tiene una manifestación fisiológica en la piel. La reacción emocional se ve reflejada en el grado de activación - transpiración de las glándulas sudoríparas exocrinas: a mayor transpiración, mayor intensidad emocional y viceversa. Resultados similares se describen en el estudio de (Mojica-Londoño, 2017), quien destaca esta técnica como una de las biométricas esenciales en las investigaciones de neuromarketing. 
Estas glándulas son controladas por el sistema nervioso simpático, integrante del sistema nervioso autónomo ${ }^{5}$ (Gallegos \& Torres, 1983). El agua es buen conductor de la electricidad, por lo tanto, con los sensores colocados en la superficie de la epidermis ${ }^{6}$, es posible registrar las variaciones emocionales experimentadas por un sujeto ante un estímulo externo. La sudoración originada por la exaltación neurovegetativa del sistema simpático, acorde a los estados de ansiedad o nerviosismo, reduce la resistencia eléctrica de la piel; en oposición, la resistencia se incrementa en los estados de sosiego y relajación. El estudio de Groeppel-Klein (2005), basado en la actividad electrodérmica y el ritmo cardiaco, manifiesto que tanto los compradores, como los no compradores que se pasean por un centro comercial tienen distintos patrones de activación. Es una métrica incorporada habitualmente a las investigaciones de neuromarketing para detectar el impulso de compra en los sujetos estudiados.

Para la publicidad de marca, la medición de la RGP, permite optimizar la estrategia de posicionamiento de marca y su diferenciación de la competencia; diseñar perfiles de clientes tarjet y nichos de mercado, calificar la huella emocional de campañas promocionales, anuncios y contenido audiovisual. Su relación con el eyetracking o seguimiento ocular, se basa en el interés empresarial de combinar ambas técnicas en el marketing digital, lo que coincide con investigaciones de Mojica-Londoño (2017). Se complementan para calcular el impacto sensorial de diferentes propuestas audiovisuales (conocer la efectividad del diseño gráfico, qué banner es más efectivo en una página web; identificar las tasas de rebote o abandono, a través de picos de estrés, cansancio ocular, confusión y repleción). El análisis de sus resultados y su representación gráfica, permite disponer de tendencias y determinar patrones de comportamiento, lo que mejora la usabilidad y la navegación por la web.

Para entender la relación del efecto placebo en el marketing, se toma como referencia su descripción en la medicina, como una respuesta biológica epontánea de una persona ante una incitación inerte de una dolencia a tratar. La mente, sujetada al placebo, moviliza el sistema psiconeuroendocrino, generando una salida fisiológica, que subsana el estado patológico (Silva Bustillos, 2017). El centro de este sistema es el subsistema límbico, encargado de las emociones. La representación de estímulos visuales en una sugestión de compra, por ejemplo, incita este sistema y determina aquellas áreas cerebrales activadas en el sujeto estudiado. Estas premisas han formado parte de ensayos comparativos de programación neurolingüística, con aplicación a sectores como la economía y el turismo.

\section{Conclusiones}

- La aplicación del Análisis multivariado mediante Clúster, con sus algoritmos correspondientes e indicadores de bondad, lograron identificar las variables de

\footnotetext{
${ }^{5}$ Encargado de la sudoración, donde interviene la neurotransmisora acetilcolina.

${ }^{6}$ Se coloca un dispositivo con dos sensores o electrodos en la mano o en el pie, por ser las partes del cuerpo con mayor densidad de glándulas sudoríparas ecrinas y donde mejor se registran los cambios en conductividad.
} 
mayor trascendencia en la producción científica internacional sobre el neuromarketing en el sector del turismo.

- A partir de su análisis correlacional, se evidencian las preeminencias de la aplicación combinada de tecnologías neurocientíficas como resonancia magnética y electroencefalograma con otras mediciones biométricas, sea el caso de la respuesta galvánica de la piel, seguimiento ocular (eye-tracking), medición del ritmo cardíaco o la electromiografía.

- Las relaciones positivas entre los descriptores objetos de estudio, abordados por una parte significativa de los autores, denota la importancia del estudio de los factores psicológicos y emocionales que determinan el comportamiento del consumidor durante el proceso de compra de un producto o servicio turístico.

\section{Referencias bibliográficas}

Brenninkmeijer, J., Schneider, T., \& Woolgar, S. (2019). Witness and Silence in Neuromarketing: Managing the Gap between Science and Its Application. Science, Technology, \& Human, 20 (10), 1-25.

Castillo, Y., \& Miranda, I. (2014). COMPAPROP: Sistema para comparación de proporciones múltiples. Revista de Protección Vegetal , 29 (3), 231-234.

Fajardo Egües, Y. (2016). Bibliometric study of the Cuban Journal of Hygiene and Epidemiology during the period 1995-2014. Bachelor Thesis, Universidad Central "Marta Abreu" de Las Villas, Faculty of Mathematic Physic and Computer Sciences, Las Villas.

Fajardo-Egües, Y. (2016). Bibliometric study of the Cuban Journal of Hygiene and Epidemiology during the period 1995-2014. Bachelor Thesis, Universidad Central "Marta Abreu" de Las Villas, Faculty of Mathematic Physic and Computer Sciences, Las Villas.

Gallegos, X., \& Torres, F. (1983). Bíorretroalimentación de la respuesta galvánica de la piel y entrenamiento en relajación: un estudio piloto. Revista Latinoamericana de Psicología. Fundación Universitaria Konrad Lorenz, 15 (1-2), 259-275.

Hair, J. F.; Anderson, R. E.; Tatham, R. L.; lack, W. C (1999). Análisis Multivariante. Practice Hall Iberia. Madrid. España. 799p.

Kohavi, R (1995). Wrappers for performance enhancement and oblivious decision graphs. Ph.D. diss., Stanford University, California, EEUU.

Martínez, F. (2018). Aplicación de técnicas de minería de datos con el software weka. España: Universidad de Salamanca.

Miranda, I (1997). Análisis de clúster como estrategia multivariada de clasificación. Solución a un problema taxonómico. Tesis para optar por el título de Master en Matemática Aplicada a las Ciencias Agropecuarias. CENSA- ISAAC. 
Mojica-Londoño, A. G. (2017). Electrodermal activity applied to psychology: bibliometric analysis. Revista Mexicana de Neurociencia , 18 (4), 46-56.

Moráguez, A (2011). ¿Cómo seleccionar el tamaño de una muestra para una investigación educacional? [en línea]. La Habana. Cuba. Disponible en: www.monografía.com. [Consulta:22 de febrero 2021].

Nonone, E., \& Regalado, M. (2019). El neuroturismo y su relación con el turismo accesible como una propuesta para la ciudad de Lima. Tesis Doctoral en Turismo, Escuela Profesional de Turismo y Hotelería. Universidad de San Martín de Porres, Facultad de Ciencias de la Comunicación, Turismo y Psicología, Lima.

Pérez, C (2018). Reglas de asociación. Aplicación práctica en la cesta de la compra de los consumidores. Trabajo en opción al grado en Marketing e Investigación de mercados. Facultad de Ciencias Económicas y Empresariales. Universidad de Sevilla. España. 58p.

Silva Bustillos, R. (2017). Programación Neurolingüística (PNL), Neuromarketing y Placebo. Recuperado el 31 de Marzo de 2021, de Repositorio Institucional de la Universidad Politécnica Salesiana: http://dspace.ups.edu.ec/handle/123456789/11019

Srikant, R y Agrawal, R (1996). Mining quantitative association rules in large relational tables. AcmSigmod Record, Volume 25. ACM, 1-12.

Victoria, J., Arjona, J., \& Repiso, R. (2015). El paradigma del neuromarketing a la luz de su producción científica. Revista Venezolana de Información, Tecnología y Conocimiento , 12 (2), 26-40.

Vilà Baños, R., Rubio Hurtado, M. J., Berlanga Silvente, V., \& Torrado Fonseca, M ( 2014). Cómo aplicar un cluster jerárquico en SPSS. Obtenido de Reire. Revista d’Innovació i Recerca en Educació: http://www.ub.edu/ice/reire.htm

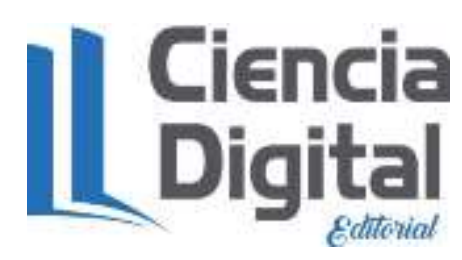




\section{PARA CITAR EL ARTÍCULO INDEXADO.}

Méndez Lazo, S. R., Vázquez Alfonso, Y., Lazo del Vallín, S., \& Carrasco Ruano, Y. T. (2021). Estudio de investigaciones sobre el neuromarketing en turismo. Visionario Digital, 5(4), 30-41. https://doi.org/10.33262/visionariodigital.v5i4.1876

\section{Liencia}

El artículo que se publica es de exclusiva responsabilidad de los autores y no necesariamente reflejan el pensamiento de la Revista Visionario Digital.

El artículo queda en propiedad de la revista y, por tanto, su publicación parcial y/o total en otro medio tiene que ser autorizado por el director de la Revista Visionario Digital.
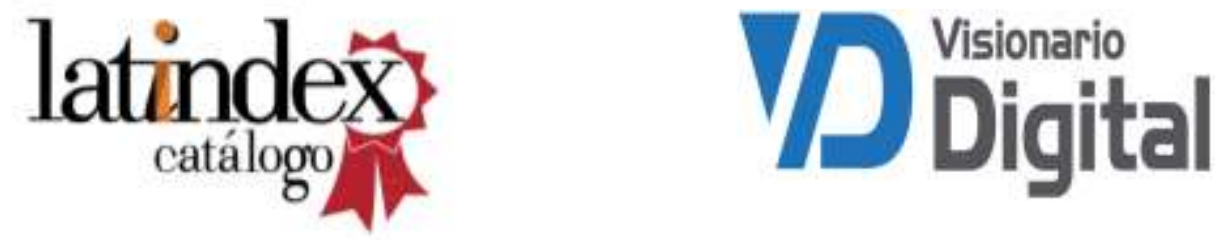